\title{
(Not so) hierarchical stellar multiples seen through the eyes of Kepler, TESS, and other missions
}

\author{
T. Borkovits ${ }^{1,2}$ and S.A. Rappaport ${ }^{3}$ \\ 1 Baja Astronomical Observatory of Szeged University, Hungary \\ 2 Konkoly Observatory, Hungary \\ 3 Department of Physics, Kavli Institute for Astrophysics and Space Research, \\ M.I.T., Cambridge, MA 02139, USA
}

Received: November 2, 2019; Accepted: November 11, 2019

\begin{abstract}
During the era of Kepler, TESS, and other related star-monitoring space-based missions, quasi-continuous observations of thousands of known and previously unknown eclipsing binaries for several months (or even years) has led to the discovery of hundreds of compact hierarchical triple (and multiple) star systems. Many of them produce spectacular observational effects that were never (or at least, rarely) seen before, for example: extra outer eclipses; thirdbody perturbation-dominated, large amplitude, non-sinusoidal eclipse timing variations; rapid eclipse depth variations, etc. Successful modeling of these phenomena is a great challenge; however, it does offer substantial astrophysical benefits. In this paper we review our two different approaches to these challenges: one of them is based on the analytical theory of third-body perturbations and is applied exclusively to the eclipse timing variations, while the other is a complex, numerical, spectro-photodynamical modeling of all the available observations of such systems. We discuss some recent results obtained for TESS systems.
\end{abstract}

Key words: binary and multiple stars - eclipsing binaries - celestial mechanics

\section{Introduction}

Past and recently operating space telescopes dedicated to searching for transiting extrasolar planets have been producing unprecedentedly high quality and long-term lightcurves of thousands of eclipsing binaries (EBs) for more than a decade. Many of these EBs have been found to be members of compact, hierarchical triple (CHT) stellar systems. A significant fraction of these triples are so compact, that the third component perturbs the motion of the EB which leads to eclipse timing variations (ETV) having a characteristic period of $P_{2}$ (i. e., the orbital period of the outer component) with an amplitude that can exceed that of the classic, well-known light travel-time effect by huge factors. Furthermore, these ETVs may also be accompanied by dramatic eclipse depth 
variations and/or extremely fast (sometimes retrograde) apsidal motion. A more spectacular and rare phenomenon observed for the first time by the Kepler mission are the extra eclipses in about a dozen CHTs (as well as a similar number of circumbinary planets), i.e., such dimmings occur when the outer component either eclipses one or both members of the inner binary or, is eclipsed by them.

The modeling of both the dynamical ETV curves and the light variations of systems with outer eclipses need special care, but offer substantial improvements in understanding the physics of binary and multiple stellar systems. Here we refer mainly for the accurate determination of the relative inclinations of the orbital planes of the inner and outer subsystem(s) which is a key parameter from the point of view of binary and multiple star formation and evolution (see e. g. Moe \& Kratter, 2018, and further references thereins).

In this paper we discuss two different treatments developed by our groups to investigate these extraordinary phenomena.

\section{Analytic ETV model and its applications}

Three-body perturbations in a CHT are effective on three different timescales. The lowest amplitude ones have the shortest characteristic period on the order of the inner binary's period $\left(P_{1}\right)$. The middle ones (both in amplitude and period) are effective on the timescale of the third body's orbital period $\left(P_{2}\right)$, while the largest amplitude effects have a substantially longer timescale of $P_{2}^{2} / P_{1}$. While the first kind of perturbation usually remains below the limit of current detectability, and the largest ones, in general, become effective only over decades or even centuries, the middle ones may alter the ETVs and lightcurves of EBs orbiting in CHTs within months. This fact offers a good chance to determine accurate system parameters on these relatively short timescales.

The first efforts toward analytical modeling of dynamically perturbed ETVs of EBs were undertaken by Soderhjelm (1975) and Mayer (1990). The most detailed model, however, has been developed only recently by Borkovits et al. (2015). In the same paper the authors also describe their software package for solving the inverse problem. This treatment enables one to mine all the orbital elements of both the inner and outer orbits, including angular elements relative to both the tangential plane of the sky and the invariable plane of the system and, therefore, to obtain such dynamically and evolutionary important parameters as the relative inclination of the inner and outer orbits and the arguments of periastrons in the dynamical frame of reference. Moreover, in theory, physical masses may also be obtained, however, in most cases only with lower accuracy.

This approach was successfully applied to more than 60 CHTs in the original Kepler field (Borkovits et al., 2015, 2016). Later it was also applied to other triple system candidates observed by the CoRoT satellite (Hajdu et al., 2017) and K2 mission (Borkovits et al., 2019a,b). Most recently, the ETVs of the first 
two CHTs measured by the TESS spacecraft are also under analysis (Borkovits et al., 2020, in preparation). Illustrations are shown in Fig. 1.
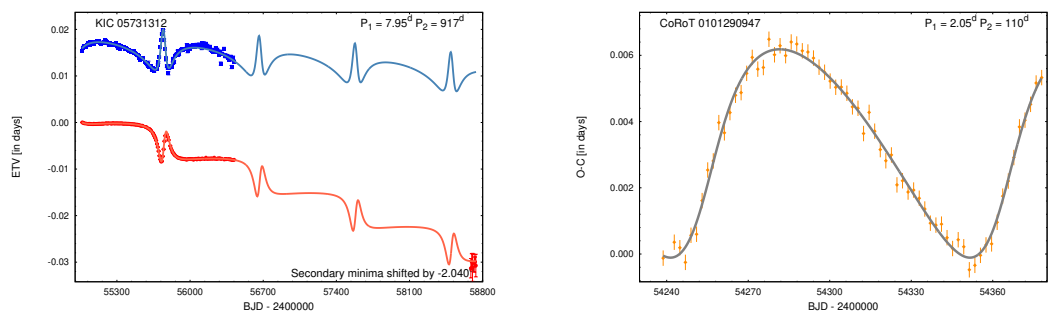

Figure 1. Dynamical ETV solutions for KIC 5731312 (left) and CoRoT 101290947 (right). For the Kepler system recent TESS observations are also included. Red points stand for primary eclipses, while blue boxes represent secondary ones. (For the epoch of the TESS measurements, the secondary eclipses have already disappeared due to the rapid precession of the orbital plane.)

\section{A complex spectro-photodynamical approach in the mod- elling of different types of observational input data}

While dynamical ETV analyses offer quick results for a large sample of CHTs, it omits a significant amount of other available relevant information which can be obtained from the same lightcurve observations (i.e., eclipse duration and depth variations, possible extra eclipses), not to mention other kinds of measurements such as, e.g., radial velocity (RV) observations, and spectral energy ('SED') measurements. In order to take into account all the available information we have initiated the development of a software package which allows joint, complex analyses of multi-band lightcurves, RV and ETV curves of binary, hierarchical triple and quadruple (both $2+2$ and $3+1$ hierarchies) systems. The orbital motions either be taken to be unperturbed Keplerian orbits or, can be calculated via numerical integration. Triple and multiple eclipses are also modeled. Moreover, a joint analysis including the SED of the two, three or four stars, as well as the optional use of PARSEC stellar isochrones (Bressan et al., 2012) have also been incorporated (see Borkovits et al., 2019b, and further references therein). Note, other recently developed software packages offer partly or fully similar services (see, e.g. Prša, 2018, and further references therein).

Most recently our software package has been used for the analysis of two CHTs discovered with TESS in and near to its southern continuous viewing zone. We identified both TICs 167692429 and 220397947 through their rapidly varying ETVs showing clear signs of the perturbations of a third stellar component. Furthermore, the former EB also manifests continuous eclipse depth variations 
and a clear feature (both in the timing and depth variations) of the periastron passage of a remarkably eccentric, and inclined third star.

Our analyses have revealed that both systems consist of inner eclipsing pairs of metal deficient, probably old F-type twin stars and less massive, late $\mathrm{K}$ or, early M-type outer components. In the case of TIC $1692429\left(P_{1}=10.26 \mathrm{~d}\right.$, $\left.P_{2}=331\right)$ both orbits have significant eccentricity $\left(e_{1}=0.17, e_{2}=0.56\right)$ and the relative inclination is $i_{\text {rel }}=27^{\circ}$ which results in orbital plane precession with a period of $P_{\text {node }} \approx 70 \mathrm{yr}$. Within one precession cycle the inner pair exhibits eclipses during two $\sim 10-11$ yr-long intervals. Moreover, we found, that during one third of a precession cycle the system is expected to show outer eclipses. TIC 220397947 , the tighter of the two systems $\left(P_{1}=3.56 \mathrm{~d}, P_{2}=77 \mathrm{~d}\right)$ was found to be perfectly flat $\left(i_{\text {rel }} \sim 0.5^{\circ}\right)$; however, there are some signs suggesting that the system might consist of a fourth star, too, revolving on an orbit having a period of $P_{3} \sim 2700 \mathrm{~d}$ and relative inclination $i_{\text {rel2 }} \sim 10-20^{\circ}$. Detailed analysis of these systems will be published in the near future.

Acknowledgements. This work has been supported by the NKFIH Grant KH130372 of Hungarian National Research, Development and Innovation Office.

\section{References}

Borkovits, T., Hajdu, T., Sztakovics, J., et al., A comprehensive study of the Kepler triples via eclipse timing. 2016, Mon. Not. R. Astron. Soc., 455, 4136, DOI: $10.1093 / \mathrm{mnras} / \mathrm{stv} 2530$

Borkovits, T., Rappaport, S., Hajdu, T., \& Sztakovics, J., Eclipse timing variation analyses of eccentric binaries with close tertiaries in the Kepler field. 2015, Mon. Not. R. Astron. Soc., 448, 946, DOI: 10.1093/mnras/stv015

Borkovits, T., Rappaport, S., Kaye, T., et al., Photodynamical analysis of the triply eclipsing hierarchical triple system EPIC 249432662. 2019a, Mon. Not. R. Astron. Soc., 483, 1934, DOI: 10.1093/mnras/sty3157

Borkovits, T., Sperauskas, J., Tokovinin, A., et al., The compact multiple system HIP 41431. 2019b, Mon. Not. R. Astron. Soc., 487, 4631, DOI: 10.1093/mnras/stz1510

Bressan, A., Marigo, P., Girardi, L., et al., PARSEC: stellar tracks and isochrones with the PAdova and TRieste Stellar Evolution Code. 2012, Mon. Not. R. Astron. Soc., 427, 127, DOI: 10.1111/j.1365-2966.2012.21948.x

Hajdu, T., Borkovits, T., Forgács-Dajka, E., et al., A search for tight hierarchical triple systems amongst the eclipsing binaries in the CoRoT fields. 2017, Mon. Not. R. Astron. Soc., 471, 1230, DOI: 10.1093/mnras/stx1523

Mayer, P., Eclipsing Binaries with Light-time Effect. 1990, Bulletin of the Astronomical Institutes of Czechoslovakia, 41, 231

Moe, M. \& Kratter, K. M., Dynamical Formation of Close Binaries during the Premain-sequence Phase. 2018, Astrophys. J., 854, 44, DOI: 10.3847/1538-4357/aaa6d2 
Prša, A. 2018, Modeling and Analysis of Eclipsing Binary Stars; The theory and design principles of PHOEBE

Soderhjelm, S., The three-body problem and eclipsing binaries. Application to Algol and lambda Tauri. 1975, Astron. Astrophys., 42, 229 